\title{
Physiology-Based MR Imaging Assessment of CSF Flow at the Foramen Magnum with a Valsalva Maneuver
}

\author{
R.A. Bhadelia, N. Madan, Y. Zhao, M.E. Wagshul, C. Heilman, J.P. Butler, and S. Patz
}

\begin{abstract}
BACKGROUND AND PURPOSE: MR imaging is currently not used to evaluate CSF flow changes due to short-lasting physiological maneuvers. The purpose of this study was to evaluate the ability of MR imaging to assess the CSF flow response to a Valsalva maneuver in healthy participants.
\end{abstract}

MATERIALS AND METHODS: A cardiac-gated fast cine-PC sequence with $\leq 15$-second acquisition time was used to assess CSF flow in 8 healthy participants at the foramen magnum at rest, during, and immediately after a controlled Valsalva maneuver. CSF mean displacement volume $\bar{V}_{C S F}$ during the cardiac cycle and CSF flow waveform $A_{p p}$ were determined. A work-in-progress real-time pencil-beam imaging method with temporal resolution $\leq 56$ ms was used to scan 2 participants for 90 seconds during which resting, Valsalva, and post-Valsalva CSF flow, respiration, and HR were continuously recorded. Results were qualitatively compared with invasive craniospinal differential pressure measurements from the literature.

RESULTS: Both methods showed 1) a decrease from baseline in $\bar{V}_{C S F}$ and $A_{p p}$ during Valsalva and 2) an increase in $\bar{V}_{C S F}$ and $A_{p p}$ immediately after Valsalva compared with values measured both at rest and during Valsalva. Whereas fast cine-PC produced a single CSF flow waveform that is an average over many cardiac cycles, pencil-beam imaging depicted waveforms for each heartbeat and was able to capture many dynamic features of CSF flow, including transients synchronized with the Valsalva maneuver.

CONCLUSIONS: Both fast cine-PC and pencil-beam imaging demonstrated expected changes in CSF flow with Valsalva maneuver in healthy participants. The real-time capability of pencil-beam imaging may be necessary to detect Valsalva-related transient CSF flow obstruction in patients with pathologic conditions such as Chiari I malformation.

ABBREVIATIONS: $A_{P D}=C S F$ flow waveform peak-to-peak amplitude; cine-PC $=$ cine phase-contrast; $\mathrm{HR}=$ heart rate; $\mathrm{PBI}=$ pencil-beam imaging; $\bar{V}_{C S F}=\mathrm{CSF}$ mean displacement volume

nvasive pressure studies have shown that physiologic challenges such as the Valsalva maneuver or coughing produce transient changes in intracranial and intraspinal pressures. ${ }^{1}$ Although such transients are rarely of significance in healthy participants, for

Received November 7, 2012; accepted after revision December 18.

From the Department of Radiology (R.A.B.), Beth Israel Deaconess Medical Center, Boston, Massachusetts; Departments of Radiology (N.M.) and Neurosurgery (C.H.), Tufts Medical Center, Boston, Massachusetts; Phillips Medical Systems (Y.Z.), Boston, Massachusetts; Gruss MR Research Center and Department of Radiology and Biophysics (M.E.W.), Albert Einstein College of Medicine, Bronx, New York; Department of Environmental Health (J.P.B.), Harvard School of Public Health and Department of Medicine, Harvard Medical School, Boston, Massachusetts; and Department of Radiology (S.P.), Brigham and Women's Hospital, Boston, Massachusetts.

This study was supported by a grant from the Conquer Chiari Foundation.

Please address correspondence to Rafeeque A. Bhadelia, MD, Department of Radiology, Beth Israel Deaconess Medical Center, WCB90, 330 Brookline Ave, Boston, MA 02115; e-mail: rbhadeli@bidmc.harvard.edu

$\checkmark$ indicates article with supplemental on-line figure.

http://dx.doi.org/10.3174/ajnr.A3509 patients with foramen magnum obstruction such as Chiari I malformation, these physiologic alterations may be responsible for producing headache and syringomyelia. ${ }^{2-5}$ In the past, attempts were made to use CSF pressure measurements with physiologic challenges as a clinical test. ${ }^{4,6}$ However, these methods have not gained widespread acceptance because they are invasive. Although noninvasive assessment of CSF flow pulsations by MR imaging that measure bidirectional CSF flow from pressure changes between the head and spine during a cardiac cycle ${ }^{7,8}$ can be used as a surrogate, all but one of the prior implementations of cine-PC MR imaging for CSF flow assessment required approximately 100 heartbeats, making it too long to use with a transient physiologic challenge. As a result, most cine-PC studies of CSF flow have only been used during quiet breathing, both in healthy participants and in patients with suspected CSF circulation abnormalities..$^{7-14}$ The 1 exception is a 2006 abstract by Wentland et $\mathrm{al}^{15}$ reporting CSF flow during rest and Valsalva by using an undersampled projection reconstruction method with data acquisi- 


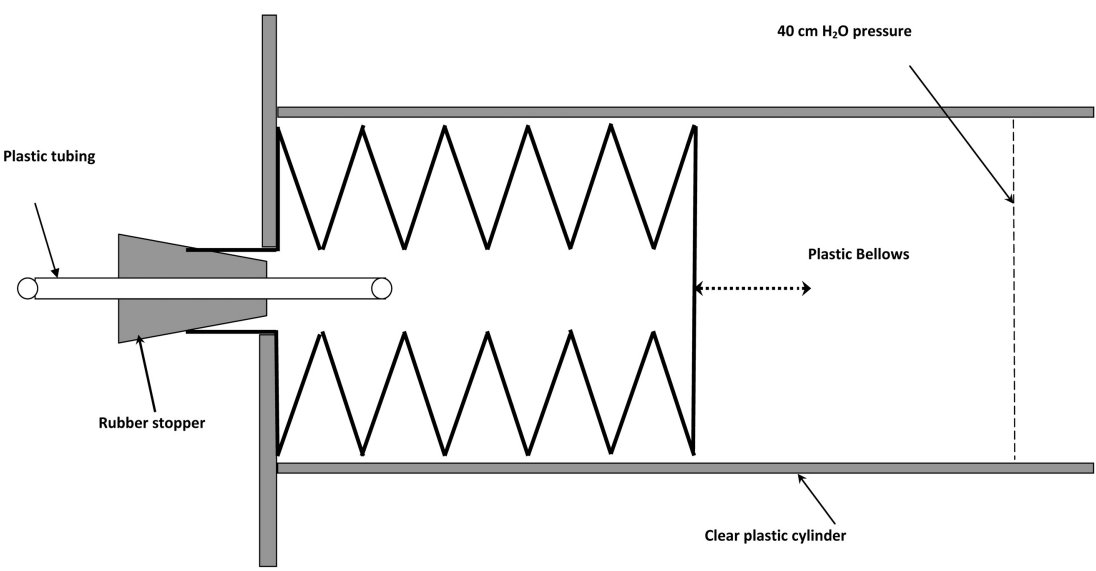

FIG 1. Schematic of Valsalva device used for experiments. Participants were directed to exhale into the plastic tubing with sufficient force to hold the end of the bellows at the $40-\mathrm{cm} \mathrm{H}_{2} \mathrm{O}$ mark on the clear plastic cylinder. This provided a consistent and reproducible Valsalva-generated pressure.

\section{CSF Flow-Imaging Sequences}

CSF flow pulsations were assessed with 2 different techniques. The first flow-imaging technique was a fast cine-PC sequence that was achieved by use of a combination of parallel imaging (sensitivity encoding factor $=2.1$ ), half Fourier acquisition (half-Fourier factor $=$ 0.6 ), and multiple-phase encodes per cardiac phase (turbo field-echo factor $=$ $3)$. Other imaging parameters were $T R$, $11 \mathrm{~ms}$; TE, $7.3 \mathrm{~ms}$; field of view, $15 \times 15$ $\mathrm{cm}$; and voxel size, $1 \times 1 \times 5 \mathrm{~mm}$. The velocity encoding was performed at 5 $\mathrm{cm} / \mathrm{s}$ in all participants. This was repeated at $7 \mathrm{~cm} / \mathrm{s}$ in a single patient because of aliasing. The sequence was retrospectively gated to the heartbeat via electrocardiographic leads.

tion times of as little as 16 heartbeats. For healthy participants, however, only minimal differences in flow were seen.

Our purpose was to evaluate the ability of MR imaging to assess the CSF flow response to a Valsalva maneuver. To determine this, we studied healthy participants and compared the assessed CSF flow response with that which can be inferred from known invasive craniospinal differential pressure measurements in healthy participants. ${ }^{1}$

At first, we used a standard 2D cine-PC method with phase encoding and with a combination of undersampling and acceleration techniques to produce CSF flow images in approximately 15 seconds. We used this fast $2 \mathrm{D}$ cine-PC method to measure CSF flow before, during, and after a Valsalva maneuver. Even with these improvements in speed, the resultant images of CSF flow as a function of heart phase are nonetheless weighted averages of the phasic behavior over all of the cardiac cycles during the roughly 15 -second data acquisition. Thus, the cine-PC method does not capture heartbeat-to-heartbeat transient behavior during the Valsalva maneuver, as demonstrated in the invasive pressure measurements of Williams. ${ }^{1}$ Therefore, we also investigated a second method, that is, a work-in-progress real-time 1D PBI method with a temporal resolution of $\leq 56 \mathrm{~ms}$. Cine-PC has superior spatial resolution compared with $\mathrm{PBI}$ but with a trade-off of longer data acquisition time than PBI. Because these 2 methods are quite different, they do not lend themselves to direct comparison. Therefore, we report here on the strengths and weaknesses of each in the evaluation of Valsalva-related changes in CSF flow at the foramen magnum.

\section{MATERIALS AND METHODS}

Nine healthy participants (mean age, $34.0 \pm 11.1$ years; 4 women) underwent MR imaging on a 3T scanner (Achieva; Philips Healthcare, Best, the Netherlands). Institutional review board approval was obtained for this Health Insurance Portability and Accountability Act-compliant prospective study, and each participant signed an informed consent. None of the participants had a history of cardiovascular or neurologic disorders.
The second flow-imaging technique was PBI. This pulse sequence excites a narrow cylinder or "pencil" region and has been described in detail previously. ${ }^{16,17}$ Because this sequence is not available for routine clinical use, a research version was used. The sequence consisted of a nominal 20-mm diameter cylindrical excitation by using a $2 \mathrm{D}$ spatially localized radio-frequency pulse with 8-turn spiral gradients, followed by a bipolar velocity-encoding gradient and a readout gradient applied along the axis of the cylinder (pencil beam). PBI thus provides spatial resolution only along the axis of the cylinder. The pencil beam was $64 \mathrm{~mm}$ in length, with a velocity encoding of $5 \mathrm{~cm} / \mathrm{s}$ along the superior to inferior direction. Other imaging parameters were TR, 25-28 ms; TE, $3.8 \mathrm{~ms}$; and flip angle, $10^{\circ}$. The bipolar phaseencoding gradient was alternated on sequential TRs, making the effective temporal resolution $2 \times$ TR (ie, 50-56 ms). No gating was used, but HR (from the electrocardiogram) and respiration (from the respiratory bellows) were continuously monitored by using the scanner's physiologic recording system.

\section{Confirmation of Bipolar Phase-Encoding Gradient Velocity Encoding}

Using a MR imaging compatible flow pump (Compuflow 1000; Shelly Medical Technologies, Ontario, Canada), we performed a phantom study. The pump was programmed to provide waveforms with various shapes, flow rates, and amplitudes (in the physiologic range of CSF flow rates and amplitudes). The MR-measured flow in the phantom experiments was in excellent agreement $( \pm 5 \%)$ with the actual flow programmed in the pump.

\section{Valsalva Maneuver}

A nonmagnetic mechanical bellows device was designed and built to perform a controlled Valsalva maneuver in the MR imaging environment (Fig 1). It consists of flexible plastic bellows tubing closed at 1 end, and with the extension of the closed end calibrated for pressures ranging from $20-40 \mathrm{~cm} \mathrm{H}_{2} \mathrm{O}$. To perform a Valsalva maneuver, a participant was asked to blow into the tube until the pressure reached a target pressure, typically $40 \mathrm{~cm} \mathrm{H}_{2} \mathrm{O}$, indicated by the bellows extension to a mark on the cylinder. The participant was asked to 


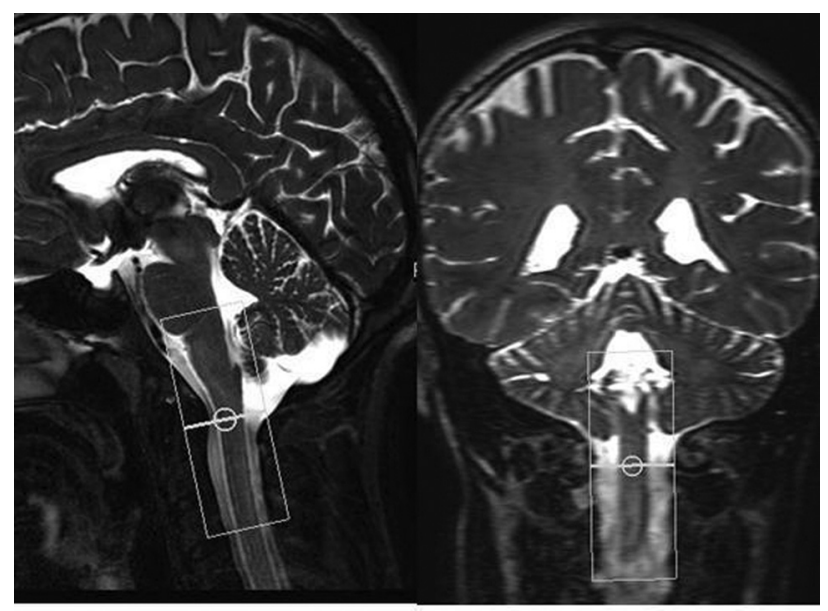

FIG 2. Sagittal and coronal T2-weighted images show PBI excitation cylinder extending from above the level of the foramen magnum to the C2-3 disk level.

continue to hold the bellows extension (and hence the pressure) in the tube for the duration of the Valsalva maneuver, typically 15-20 seconds. The Valsalva device was placed on the patient bed between the participant's legs, allowing the participant (via a mirror) and the operator to observe the airway pressure.

\section{MR Imaging}

Sagittal T1- or T2-weighted images were obtained to provide anatomic details. A total of 9 participants underwent scanning, along with use of the Valsalva maneuver. Before the availability of PBI, 7 participants were studied by use of fast cine-PC. The eighth participant underwent scanning with both fast cine-PC and PBI, and the ninth participant underwent scanning with PBI alone.

Fast cine-PC scans were performed in the axial plane at the level of the posterior arch of the first cervical vertebra (to avoid including the vertebral arteries in the imaging plane). Initially, the participant was asked to breathe quietly, and a resting scan was performed. The participant was then asked to blow into the tube and maintain a target pressure. Within a few seconds of the start of the Valsalva maneuver, a second cine-PC scan was obtained. A post-Valsalva cine-PC scan was then obtained approximately 5 seconds after the participant returned to quiet breathing. The resting, Valsalva, and post-Valsalva scans were repeated 3 times. The scans were also repeated if scanning stopped during acquisition because of interruption in gating from sudden and large changes in HR during the Valsalva maneuver. Mean HR during each scan was calculated and saved in the image header.

For PBI, the pencil beam was positioned along the cervical spinal canal extending from just above the level of the foramen magnum to the C2-3 disk level (Fig 2). Serial PBI scans were continuously acquired for approximately 90 seconds in duration. For each set of scans, the participant was asked 1) to breathe quietly for the first 15-20 seconds (by counting from 1-20), 2) to then start blowing into the tube to perform the Valsalva maneuver for a similar duration, and 3 ) to breathe quietly again after the end of Valsalva. Each set of PBI scans was repeated 3 times.

\section{Image Analysis}

QFLOW software (Phillips) was used to analyze the cine-PC data. Analysis of resting, Valsalva, and post-Valsalva images was performed by drawing a region of interest around the thecal sac. Output measures were flow rate (average velocity in the region of interest times its cross-sectional area), mean CSF displacement volume $\bar{V}_{C S F}$ during the cardiac cycle (defined as the average of the absolute values of systolic and diastolic displacement volumes), and oscillatory $A_{p p}$ (sum of the absolute value of the maximal systolic and diastolic flow rates).

PBI data were analyzed by custom software written in Matlab (MathWorks, Natick, Massachusetts). CSF flow waveforms of average velocity (ie, averaged over the cross-section of the pencil beam) at each position along the PBI cylinder were plotted as a function of time, along with synchronously acquired HR and respiration data. To determine $\bar{V}_{C S F}$ from PBI, average velocity was multiplied by the cross-sectional area of the thecal sac, which was determined by using an independent anatomic scan.

\section{Data Analysis}

For each participant studied with cine-PC, values of $\bar{V}_{C S F}, A_{p p}$, and HR from 3 experiments were averaged. Using a paired $t$ test, all 3 parameters were compared between resting vs Valsalva, Valsalva vs post-Valsalva, and resting vs post-Valsalva periods. All statistical analyses were performed with SPSS software (SPSS, Chicago, Illinois), with $P<.05$ considered significant. Because only 2 participants underwent scanning with PBI, statistical analyses were not performed. For these participants, the measured real-time CSF flow, $\mathrm{HR}$, and respiration for the entire 90-second data acquisition that comprised the 3 periods are reported. To interpret these results, we evaluated them in qualitative comparison with craniospinal differential pressure measurements from the literature. ${ }^{1}$

\section{RESULTS}

\section{Fast Cine-PC}

All participants were able to undergo scanning without difficulty. Measured values of $\bar{V}_{C S F}, A_{p p}$, and HR during the 3 different physiologic states are given in Table 1. CSF flow from 1 participant is shown in Fig 3. A decrease in both CSF flow parameters and an increase in HR were observed during Valsalva compared with the resting values. An increase in both CSF flow parameters and a decrease in HR were seen in post-Valsalva compared with during Valsalva. Finally, when comparing values in the post-Valsalva period with resting conditions, both $\bar{V}_{C S F}$ and $A_{p p}$ were significantly higher, indicating a post-Valsalva rebound effect, but changes in HR were not statistically significant (Table 1).

\section{Pencil-Beam Imaging}

The On-line figure shows PBI results in the 2 participants studied. Although PBI can display flow at various positions along the 6.4$\mathrm{cm}$-long excitation cylinder, we focused on measurements restricted to the level of the $\mathrm{C} 1$ vertebra for this preliminary study. Although in both participants, changes in bidirectional CSF flow during the resting, Valsalva, and post-Valsalva periods were similar to those observed with fast cine-PC, simultaneous real-time display of respiration made it straightforward to observe the pre- 
Table 1: CSF flow displacement volume per cardiac cycle, peak-to-peak amplitude, and heart rate at rest, during, and post-Valsalva maneuver, from fast cine-PC measurements

\begin{tabular}{lcccccc}
\hline & & & & \multicolumn{2}{c}{ Comparison (P Value) } \\
\cline { 3 - 6 } & R & VAL & P-VAL & R vs VAL & VAL vs P-VAL & R vs P-VAL \\
\hline Mean CSF $\bar{V}_{C S F}(\mathrm{~mL})$ per cardiac cycle & $0.57 \pm 0.24$ & $0.37 \pm 0.17$ & $0.68 \pm 0.19$ & $<.001$ & $<.001$ & $<.05$ \\
$A_{p p}(\mathrm{~mL} / \mathrm{s})$ & $4.51 \pm 1.61$ & $3.52 \pm 1.59$ & $5.28 \pm 1.49$ & $<.007$ & $<.001$ & $\leq .01$ \\
Heart rate (beats/min) & $67.1 \pm 10.4$ & $78.5 \pm 9.9$ & $64.22 \pm 6.6$ & $<.002$ & $<.001$ & $\mathrm{NS}$ \\
\hline
\end{tabular}

NS indicates not significant; P-VAL, post-Valsalva; R, resting; VAL, Valsalva.

Note:- All values are expressed as mean \pm SD.

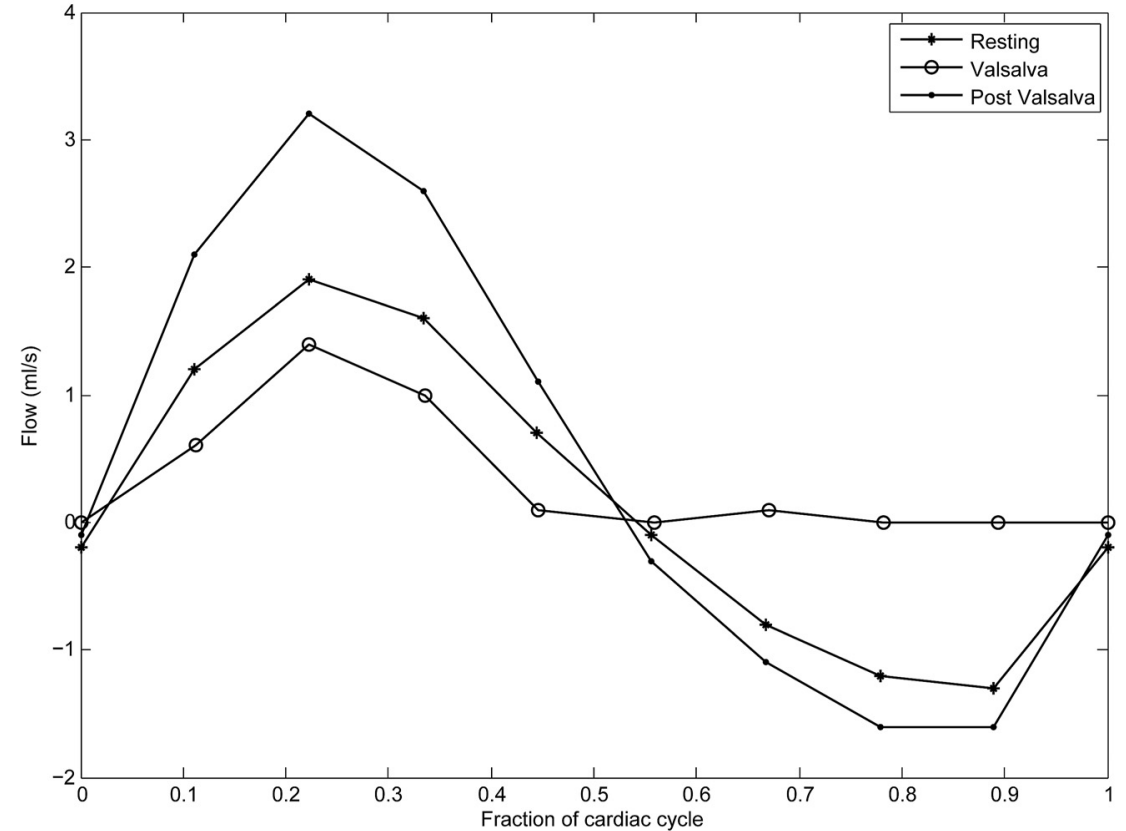

FIG 3. Cardiac cycle-dependent CSF flow before, during, and after a Valsalva maneuver in 1 participant by using fast cine-PC imaging. Flow in milliliters per second is shown as a function of time, normalized to the cardiac cycle. During Valsalva, $\bar{V}_{C S F}$ and $A_{p p}$ decreased compared with resting. After Valsalva, both $\bar{V}_{C S F}$ and $A_{p p}$ increased compared with during the maneuver, rebounding to values larger than resting.

cise timing of the Valsalva as well as the coincident heartbeat-toheartbeat variations in both $\bar{V}_{C S F}$ and $A_{p p}$.

\section{Qualitative Comparison with Previous Invasive Pressure Measurements}

Both flow-imaging methods showed a CSF flow response to Valsalva consistent with invasive craniospinal differential pressure measurements by Williams, ${ }^{1}$ (Fig 4, trace 4). The advantage of measuring CSF flow in real-time with PBI compared with fast cine-PC can be most appreciated by comparing the On-line figure with Fig 4. Here, similarities between transient changes in CSF flow waveforms during the resting, Valsalva, and post-Valsalva periods and the craniospinal differential pressure tracing (trace 4) are most apparent.

\section{DISCUSSION}

The results show that both fast cine-PC and PBI can be used to assess changes in CSF flow in response to a physiologic challenge, and lead to objective analysis of differences in response. CSF flow between the head and the spine decreases during Valsalva and increases past the resting flow rate in the post-Valsalva period. These findings are consistent with previous experiments performed by Williams ${ }^{1}$ by using simultaneous invasive measure- ments of CSF pressure in the head and spine. Table 2 shows the expected respiratory and cardiovascular changes as well as expected changes in intracranial and spinal pressure and CSF flow in 4 phases of the Valsalva maneuver. ${ }^{1,18}$ During performance of the Valsalva maneuver (phases I-II), spinal CSF pressure increases because of transmission of high intrathoracic pressure and consequent distension of the epidural veins. ${ }^{1}$ The intracranial pressure also increases, but this increase is much less because of the associated decrease in cardiac output and reduced systolic arterial inflow to the head. ${ }^{1,18}$ The higher spinal pressure relative to intracranial pressure reduces CSF flow across the foramen magnum. Immediately after normal breathing resumes (phases IIIIV), spinal pressure decreases because of reduction in intrathoracic pressure and collapse of the epidural veins. However, increased cardiac output and cranial arterial inflow momentarily increase intracranial pressure above resting value, resulting in the post-Valsalva rebound. ${ }^{1}$ The higher intracranial pressure relative to spinal pressure increases CSF flow across the foramen magnum.

Although fast cine-PC was able to show changes in CSF flow averaged during its 15-second acquisition time, PBI captured many dynamic features of CSF flow, including transients synchronized with the beginning and end of the Valsalva. In addition, our PBI results show that it is possible to noninvasively demonstrate heartbeat-to-heartbeat changes in CSF flow induced by differences in craniospinal pressure during Valsalva. ${ }^{1}$ This finding is evident by comparing CSF flow changes seen in the On-line Figure to invasive pressure measurements of Williams ${ }^{1}$ shown in Fig 4 (trace 4 ). Specifically, the gradual decrease in pressure during the Valsalva followed by the sharp rebound in pressure fluctuation amplitude on its termination is mirrored in the flow behavior revealed by the PBI approach. This is consistent with flow, especially in its transient behavior, being driven by corresponding fluctuations in pressure in the spinal column coincident with the heartbeat. It is important to note that these transient flow changes associated with the onset and termination of the Valsalva maneuver are seen with $\mathrm{PBI}$ and not with cine-PC. Finally, unlike fast cine-PC, PBI can simultaneously assess changes both above and below the level 


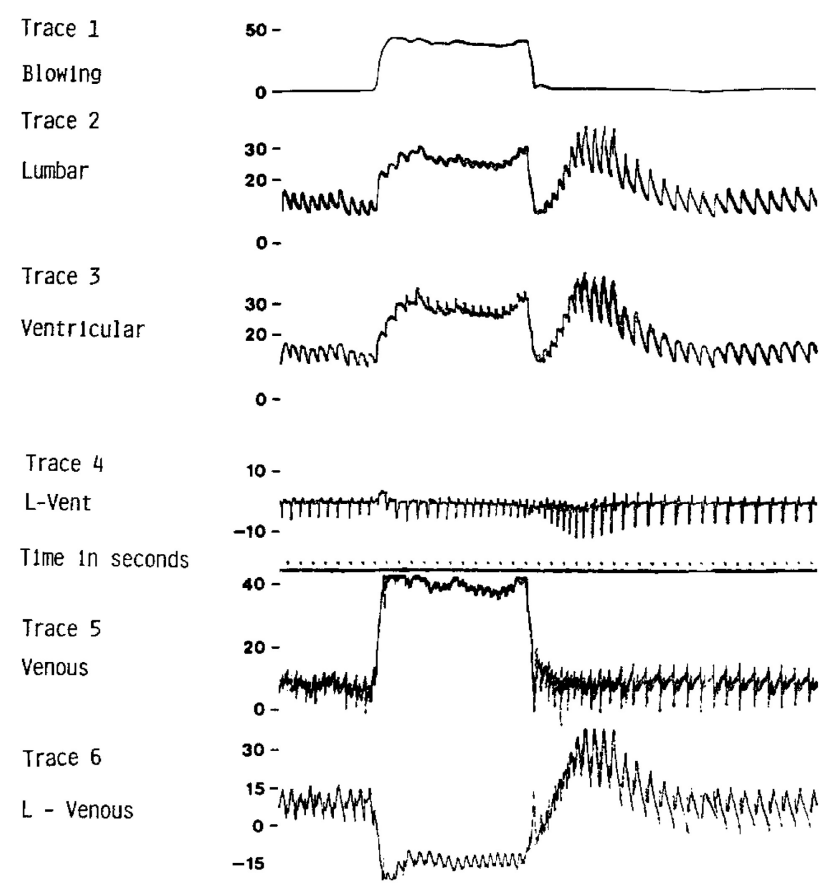

FIG 4. Figure shows invasive pressure measurements vs time. Trace 4 shows heartbeat-by-heartbeat changes in lumbar minus ventricular pressure (craniospinal pressure differential) with Valsalva. Note the similarities between trace 4 and CSF flow waveforms (panel 2) in the On-line figure. Note further that PBI captured many dynamic CSF flow features including transients precisely synchronized with timing of Valsalva similar to that seen here. Reproduced from Williams B. Simultaneous cerebral and spinal fluid pressure recordings. I. Technique, physiology, and normal results. Acta Neurochir (Wein) 1981; 58:167-85 with kind permission from Springer Science and Business Media.

of the foramen magnum, along the length of the excitation cylinder, and it does not require cardiac gating. This eliminates the possibility of gating interruption because of sudden changes in HR during the Valsalva maneuver (as described in the Results section). We believe that the real-time capability of PBI may be necessary to detect pathologically altered Valsalvainduced transient CSF flow changes in patients with pathologic conditions such as Chiari I malformation. PBI may also prove useful in evaluating a differential CSF flow response between pathology and health for other physiologic challenges such as cough, the Mueller maneuver, or neck flexion. Also, future work can include a Fourier analysis of the CSF flow waveform to determine changes synchronous with respiration during free breathing.

Although the physiology-based challenge approach we describe here to study alterations in CSF flow by MR imaging is not a novel idea, ${ }^{15,17,19,20}$ its potential has not been fully explored. The justification for this approach comes from numerous previous experimental studies on CSF pressure in humans that used a physiologic challenge to assess the CSF pressure dynamics. ${ }^{1,2,4,6,21} \mathrm{Be}$ cause CSF flow estimation by MR imaging is currently done in resting conditions, limited clinical usefulness has been found for quantitative CSF flow imaging in patient populations. ${ }^{12,22}$ This is likely because of a high variability in CSF flow pulsation amplitudes among patients because of differences in cerebral arterial and venous flow amplitudes, intracranial compliance, and the anatomy of CSF pathways. ${ }^{7,23,24}$ The major benefit of our approach is that by comparing cardiac cycle-related CSF flow for a participant at rest with that during and after a Valsalva maneuver, compensatory CSF flow changes can be assessed on an individualized basis, eliminating the need to compare CSF flow across participants.

Although physiology-based assessment of CSF flow may have a variety of clinical applications, we believe that the most immediate and obvious application is in the assessment of CSF flow in patients with Chiari I malformation. It is generally agreed that many of the symptoms and signs associated with Chiari I malformation are attributed to abnormal CSF circulation between the head and the spine secondary to obstruction

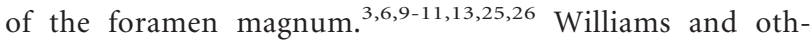
ers $^{1,4-6}$ have previously demonstrated this in patients with Chiari I malformation. On the basis of these results, we hypothesize that in patients with Chiari I malformation with a significant foramen magnum obstruction, the immediate postValsalva rebound in CSF flow shown here will be either absent or significantly reduced. We further expect that PBI will be able to observe this altered CSF flow transient and be of diagnostic value. We plan to address this hypothesis in future work.

Although we believe that PBI is superior to fast cine-PC in studying transient alterations in CSF flow in response to a physiologic challenge, it is still an emerging technology for quantitative estimation of CSF flow. As such, there are several limitations. First, the MR phase signal is not completely determined by flow within the spinal cord, as the nominal boundaries of the pencil beam may extend outside of the cord, allowing contributions from other flow sources such as the epidural veins. The shape and size of the pencil beam need to be selected so that non-CSF flow is minimally included in the PBI cross-section. Second, quantitative estimates of volume displacement similar to that obtained with cine-PC require determination of the size of the CSF pathway being assessed, which may also change during the Valsalva maneuver. This will also be the subject of future work. Finally, PBI at present is available only as a research sequence that limits its wide-

Table 2: Effects of Valsalva maneuver on intrathoracic pressure, cardiovascular system, intracranial and spinal pressures, and CSF flow

\begin{tabular}{|c|c|c|c|c|}
\hline Mechanical Effect & Phase I & Phase II & Phase III & Phase IV \\
\hline Intrathoracic pressure & $\uparrow$ & & 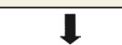 & \\
\hline Venous return & 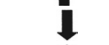 & & 1 & \\
\hline Left ventricular stroke volume & $\hat{1}$ & & 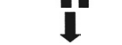 & \\
\hline Blood pressure & $\hat{1}$ & & 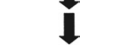 & \\
\hline Pulse rate & & & & \\
\hline Intracranial pressure & & & & \\
\hline Intraspinal pressure & & & & \\
\hline CSF flow across foramen magnum & & & & 11 \\
\hline
\end{tabular}


spread use. However, we believe that research may stimulate MR imaging vendors to make this sequence more readily available for routine clinical use.

\section{CONCLUSIONS}

Both fast cine-PC and PBI demonstrate a CSF flow response to Valsalva, consistent with previous invasive pressure measurements. Because cine-PC is a $2 \mathrm{D}$ technique, one can determine the CSF cross-sectional area allowing computation of absolute CSF flow. PBI must rely on a separate anatomic scan to translate measured average velocity over the pencil beam cross-section to flow. On the contrary, PBI captures transient changes in flow that cine-PC cannot. We believe that the real-time capability of PBI may be necessary to detect transient CSF flow changes in a pathologically altered Valsalva response in a patient population.

Disclosures: Rafeeque Bhadelia—RELATED: Grant: Conquer Chiari Foundation,* Comments: money to pay for MR imaging scans, equipment, and volunteers. Neel Madan—RELATED: Grant: Conquer Chiari Foundation, ${ }^{*}$ Comments: The institution received money for the research MR imaging scans. No money otherwise to me or my institution; UNRELATED: Consultancy: Near Infrared Imaging, Comments: Company developing a medical imaging device using photoacoustic tomography. There is no relevance to this study, other than it is in the broad category of medical imaging. Received stocks/options; Expert Testimony: expert testimony on imaging, although no cases relevant to this study. Yansong ZhaoUNRELATED: Employment: I am an employee of Philips Healthcare, Cleveland, Ohio. Mark Wagshul-UNRELATED: Payment for Lectures (including service on speaker bureaus): DoD, ${ }^{*} \mathrm{NIH} .{ }^{*}$ James Butler—RELATED: Consulting Fee or Honorarium: Beth Israel Deaconess Hospital. Samuel Patz—RELATED: Consulting Fee or Honorarium: Beth Israel Deaconess Hospital, Comments: I was paid as a Consultant Investigator for assistance with the research reported in the submitted manuscript. This also covered my assistance with data analysis and preparation of this manuscript. The Consultant fee was budgeted in a grant to Dr. Bhadelia (first author) and awarded to Beth Israel Deaconess Hospital from the Conquer Chiari Foundation. (*Money paid to institution.)

\section{REFERENCES}

1. Williams B. Simultaneous cerebral and spinal fluid pressure recordings. I. Technique, physiology, and normal results. Acta Neurochir (Wien) 1981;58:167-85

2. Heiss JD, Patronas N, DeVroom HL, et al. Elucidating the pathophysiology of syringomyelia. J Neurosurg 1999;91:553-62

3. Sansur CA, Heiss JD, DeVroom HL, et al. Pathophysiology of headache associated with cough in patients with Chiari I malformation. J Neurosurg 2003;98:453-58

4. Tachibana S, Iida H, Yada K. Significance of positive Queckenstedt test in patients with syringomyelia associated with Arnold-Chiari malformations. J Neurosurg 1992;76:67-71

5. Williams B. On the pathogenesis of syringomyelia: a review. JR Soc Med 1980;73:798-806

6. Williams B. Cough headache due to craniospinal pressure dissociation. Arch Neurol 1980;37:226-30

7. Bhadelia RA, Bogdan AR, Wolpert SM. Analysis of cerebrospinal fluid flow waveforms with gated phase-contrast MR velocity measurements. AJNR Am J Neuroradiol 1995;16:389-400

8. Enzmann DR, Pelc NJ. Normal flow patterns of intracranial and spinal cerebrospinal fluid defined with phase-contrast cine MR imaging. Radiology 1991;178:467-74
9. Alperin N, Kulkarni K, Loth F, et al. Analysis of magnetic resonance imaging-based blood and cerebrospinal fluid flow measurements in patients with Chiari I malformation: a system approach. Neurosurg Focus 2001;11:E6

10. Bhadelia RA, Bogdan AR, Wolpert SM, et al. Cerebrospinal fluid flow waveforms: analysis in patients with Chiari I malformation by means of gated phase-contrast MR imaging velocity measurements. Radiology 1995;196:195-202

11. Hofkes SK, Iskandar BJ, Turski PA, et al. Differentiation between symptomatic Chiari I malformation and asymptomatic tonsilar ectopia by using cerebrospinal fluid flow imaging: initial estimate of imaging accuracy. Radiology 2007;245:532-40

12. Krueger KD, Haughton VM, Hetzel S. Peak CSF velocities in patients with symptomatic and asymptomatic Chiari I malformation. AJNR Am J Neuroradiol 2010;31:1837-41

13. McGirt MJ, Nimjee SM, Floyd J, et al. Correlation of cerebrospinal fluid flow dynamics and headache in Chiari I malformation. $\mathrm{Neu}$ rosurgery 2005;56:716-21; discussion 716-21

14. Bhadelia RA, Frederick E, Patz S, et al. Cough-associated headache in patients with Chiari I malformation: CSF flow analysis by means of cine phase-contrast MR imaging. AJNR Am J Neuroradiol 2011;32:739-42

15. Wentland AL, Wieben O, Mistretta CA, et al. Technique for acquiring MR images of CSF flow during a Valsalva maneuver. Proc Intl Soc Mag Reson Med; 2006:1525

16. Hardy CJ, Pearlman JD, Moore JR, et al. Rapid NMR cardiography with a half-echo M-mode method. I Comput Assist Tomogr 1991; 15:868-74

17. Maier SE, Hardy CJ, Jolesz FA. Brain and cerebrospinal fluid motion: real-time quantification with M-mode MR imaging. Radiology 1994;193:477-83

18. Looga R. The Valsalva manoeuvre-cardiovascular effects and performance technique: a critical review. Respir Physiol Neurobiol 2005; 147:39-49

19. Bhadelia RA, Bogdan AR, Wolpert SM. Cerebrospinal fluid flow waveforms: effect of altered cranial venous outflow. A phase-contrast MR flow imaging study. Neuroradiology 1998;40:283-92

20. Schroth G, Klose U. Cerebrospinal fluid flow. II. Physiology of respiration-related pulsations. Neuroradiology 1992;35:10-15

21. Williams B. Simultaneous cerebral and spinal fluid pressure recordings. 2. Cerebrospinal dissociation with lesions at the foramen magnum. Acta Neurochir (Wien) 1981;59:123-42

22. Tubbs RS, Beckman J, Naftel RP, et al. Institutional experience with 500 cases of surgically treated pediatric Chiari malformation type I. J Neurosurg Pediatr 2011;7:248-56

23. Alperin N, Sivaramakrishnan A, Lichtor T. Magnetic resonance imaging-based measurements of cerebrospinal fluid and blood flow as indicators of intracranial compliance in patients with Chiari malformation. J Neurosurg 2005;103:46-52

24. Bhadelia RA, Bogdan AR, Kaplan RF, et al. Cerebrospinal fluid pulsation amplitude and its quantitative relationship to cerebral blood flow pulsations: a phase-contrast MR flow imaging study. Neuroradiology 1997;39:258-64

25. Armonda RA, Citrin CM, Foley KT, et al. Quantitative cine-mode magnetic resonance imaging of Chiari I malformations: an analysis of cerebrospinal fluid dynamics. Neurosurgery 1994;35:214-23; discussion 223-24

26. Haughton VM, Korosec FR, Medow JE, et al. Peak systolic and diastolic CSF velocity in the foramen magnum in adult patients with Chiari I malformations and in normal control participants. AJNR Am J Neuroradiol 2003;24:169-76 